Pacific Journal of Mathematics

DISCS IN COMPRESSION BODIES 


\title{
DISCS IN COMPRESSION BODIES
}

\author{
D. D. LONG
}

\begin{abstract}
It has been shown in a paper by Casson and the author that there is an algorithm to determine if an automorphism of a closed, orientable surface 'compresses'. A motivation for finding such a decision procedure is to apply it to fibred knots to determine if they are 'homotopically ribbon'. This makes it important that the process is as efficient as possible. One of the aims of this paper will be to exhibit improvements in this algorithm.
\end{abstract}

0. Introduction. We shall devote our attentions to the improvements that can be made in the case that the automorphism is pseudo-Anosov, that is, it preserves a pair of transverse, measured, singular foliations. Our results will then apply to hyperbolic knots. In particular, this simplication cut down drastically the computation of [6], in which the above methods were applied to show that the knot $14^{*}$ is not homotopically ribbon. Moreover, the method sheds some light on the way a pseudo-Anosov extends over a compression body.

The techniques we use are largely combinatorial, and exploit the special nature of the disc provided by [3] to perform traditional 3-manifold arguments.

In $\S 1$ we introduce our notation and explain the exact nature of the problem which we seek to circumvent. We may briefly explain the contents of $\$ 2$ as follows. Define a closed arc lying in a leaf of a foliation to be regular if it contains no singularities. Then one of the results of $\S 2$ is that if a pseudo-Anosov compresses, there is an embedded disc in the compression whose boundary is the union of two arcs, one of which is regular. Our main result, Theorem 2.4, is a generalization of this, and says that there is a disc whose boundary is nearly as combinatorially simple as it could be. The precise meaning of this is explained in $\S 1$.

In the course of proving 2.4 we prove the following lemma which is of interest in its own right.

Lemma 2.2. Let $\phi: F \rightarrow F$ be a pseudo-Anosov map which extends over a compression body $M$. Then there is an integer $t$ (which is bounded by some function depending only on the genus of $F$ ) and an embedded disc $D \subset M$ so that if $m$ and $n$ are any positive integers, then $\phi^{t m}(D) \cap D \cap \phi^{-t n}(D)$ can be isotoped $\operatorname{rel}(\partial M)$ so as to contain no triple points. 
This result suggests that when a pseudo-Anosov extends, it does so in a fairly 'regular' way.

In $\$ 3$ we provide some applications of the methods and results of the above. We concentrate on the case that the invariant foliations are 'globally orientable,' that is they are given by singular vector fields. For example, notice that the universal covering $p: M^{*} \rightarrow M$ induces a covering $p: F^{*} \rightarrow F$ for which $F^{*}$ is planar. Define a lifted leaf to be homoclinic if it has both ends going to the same end of $F^{*}$. Then we show:

TheOREM 3.2. Let $\phi: F \rightarrow F$ be a pseudo-Anosov with globally orientable invariant foliations $\mathscr{F}^{+}$and $\mathscr{F}^{-}$. Suppose that $\phi$ compresses in $M$. Let the induced planar covering be $p: F^{*} \rightarrow F$. Then,

(a) Both lifted foliations have a homoclinic leaf.

(b) One of the lifted foliations has at least a countable number of its leaves homoclinic.

Using 3.2 and the ideas of pleated surfaces, we show that if a pseudo-Anosov with globally orientable foliations compresses then its invariant foliations are the boundary of a collection of surfaces embedded in the compression.

1. Preliminaries. Throughout, the symbol $F$ will be reserved for a closed, orientable surface of genus at least two. A compression body for $F$ is any irreducible 3-manifold obtained from $F \times I$ by adding a nonempty set of 2- and 3-handles to $F \times(1)$. We identify $F \times(0)$ with $F$. Given an automorphism $\phi: F \rightarrow F$, we say that $\phi$ compresses (in $M$ ) if there is a compression body $M$ for $F$, and an automorphism $\Phi: M \rightarrow M$, such that $\Phi \mid F=\phi$. If $\phi$ is pseudo-Anosov, it leaves invariant a pair of transverse, measured (singular) foliations $\left(\mathscr{F}^{+}, \mu^{+}\right)$and $\left(\mathscr{F}^{-}, \mu^{-}\right)$. (For this and other standard facts concerning surface automorphisms we refer the reader to [1] and [4].) Such a pair is said to have intercept length $\leq K$ if for all arcs $\alpha^{+} \subset \mathscr{F}^{+}$, and $\alpha^{-} \subset \mathscr{F}^{-}$, both of measure $\geq K$, we have $\operatorname{int}\left(\alpha^{+}\right) \cap \operatorname{int}\left(\alpha^{-}\right)$ $\neq \varnothing$. It is easy to see that an intercept length always exists for the foliations associated with a pseudo-Anosov map.

The main result of [3] is:

Theorem A. Suppose that $\phi: F \rightarrow F$ is a pseudo-Anosov map with invariant foliations $\left(\mathscr{F}^{+}, \mu^{+}\right),\left(\mathscr{F}^{-}, \mu^{-}\right)$of intercept length $\leq K$. Further, suppose that $\phi$ compresses in $M$. 
Then there is an embedded disc $D^{2} \subset M$ with $\partial D^{2}=\alpha^{+} \cup \alpha^{-} \subset F$, where

(i) $\alpha^{+}$lies in $\mathscr{F}^{+}, \alpha^{-}$lies in $\mathscr{F}^{-}$.

(ii) $\alpha^{+}$and $\alpha^{-}$are both of measure $\leq K$.

This result provides an algorithm to determine if an automorphism compresses, for there are only finitely many simple closed curves on $F$ which satisfy (i) and (ii). Each one may be checked to see if it is a candidate for the boundary of an embedded disc in some compression of $\phi$.

However, observe that although the arcs $\alpha^{+}$and $\alpha^{-}$lie in their respective foliations, they are permitted to cut across the singularities. For example, in Figure 1, the arc $C O A$ is a permissable arc in this context. If the singularities of the foliations have order greater than 3 , this increases the length of any practical computation enormously. We aim to shed some light on this situation. First we must introduce some notation.

Recall that a separatix is half leaf with one endpoint at a singularity. We define a bent arc to be an arc which is contained in the union of two separatrices which meet at a singularity. (Observe that for the foliations which we are considering, no leaf runs from singularity to singularity; thus a bent arc contains exactly one singularity.) We include in this definition the degenerate case of an arc lying in a separatrix with one endpoint a singularity.

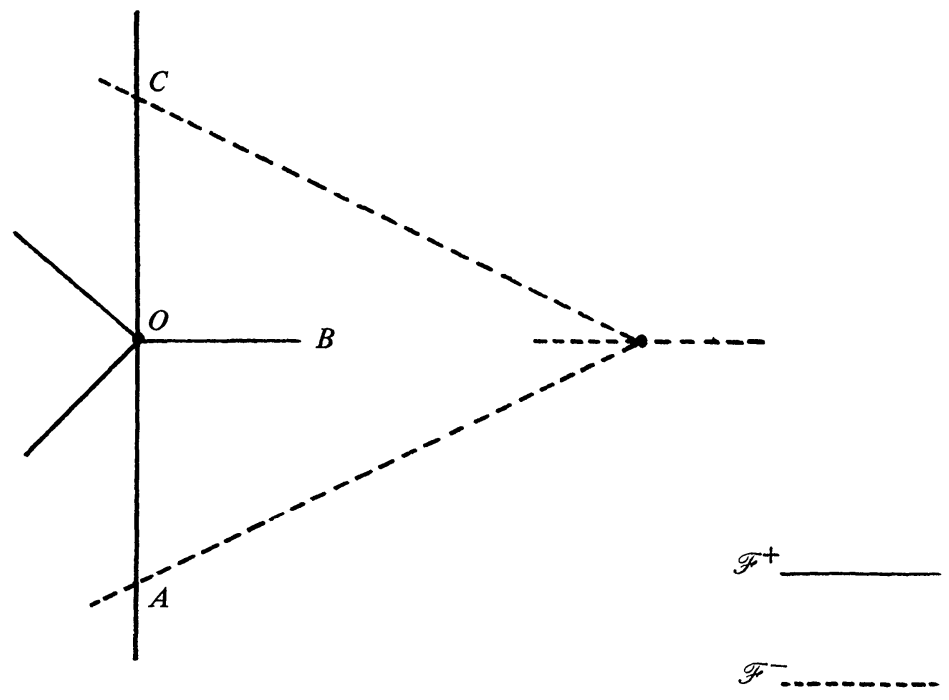

FIGURE 1 


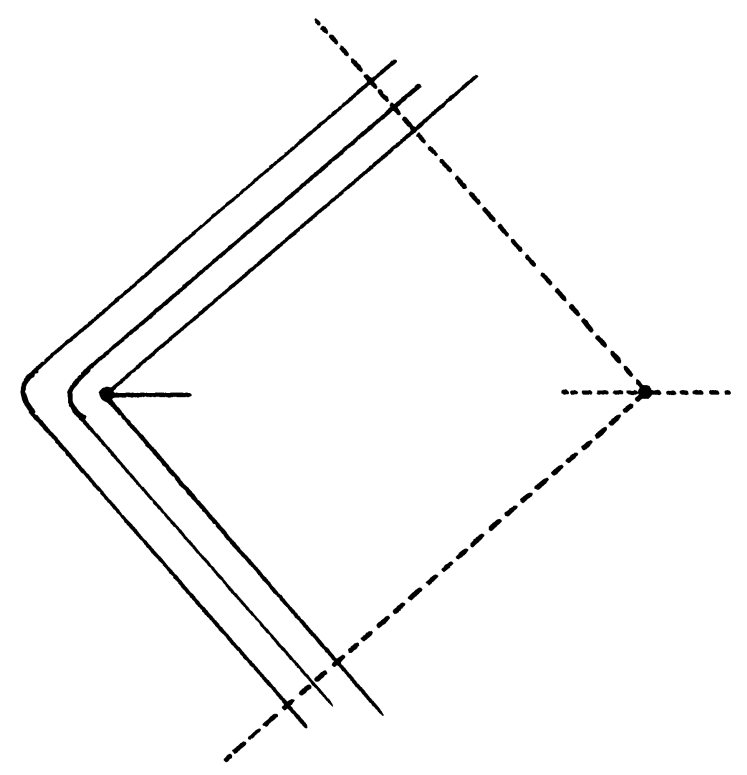

FIGURE 2

An arc is said to be regular if it lies in a leaf and has no singularity on it. Any regular arc contained in $\mathscr{F}^{+}$maybe slid transversely to $\mathscr{F}^{-}$, without changing its measure, until it lies on a singularity. See Figure 2. Thus the above theorem implies that there is an embedded disc in $M$, whose boundary is the union of two bent arcs, each of measure at most $K$. Our immediate aim is to examine the type of bent arcs which can occur.

At this point it is appropriate to note that the word 'embedded' is not strictly accurate here, since the sliding process may introduce points of self intersection at the singularities, however these are inessential in the sense that they may be removed by an arbitrarily small perturbation. The word embedded when applied to curves and discs will always be used in this sense.

Define a bent arc to be good if it is the limit of regular arcs. (Notice that the degenerate case described above is always a good bent arc.) Define the badness of a bent arc to be the smallest number of separatrices crossed in moving from the sector containing one endpoint of the arc to the sector containing the other. For example in Figure 2, the arc $C O A$ is 1-bad and the arc BOC is 0 -bad. Clearly an arc is 0-bad if and only if it is good.

We claim that in any compression there is a disc of a particularly good type. 
Theorem 2.4. Suppose that $\phi: F \rightarrow F$ is a pseudo-Anosov map with invariant foliations $\left(\mathscr{F}^{+}, \mu^{+}\right)$and $\left(\mathscr{F}^{-}, \mu^{-}\right)$of intercept length $\leq K$. Further suppose that $\phi$ compresses in $M$. Then there is an embedded disc $D^{2} \subset M$ with $\partial D^{2}=\alpha^{+} \cup \alpha^{-} \subset F$ where

(i) $\alpha^{+}$and $\alpha^{-}$are bent arcs.

(ii) $\alpha^{+}$and $\alpha^{-}$are both of measure at most $K$.

(iii) One of $\alpha^{+}, \alpha^{-}$is good, the other is at worst 1-bad.

This cuts down the length of any computation involving foliations with singularities of order greater than three drastically. Moreover, it is of some interest to know if condition (iii) is the best possible, that is, whether it can be improved to the condition that both arcs are good. This has some theoretical implications which we discuss later. At any rate, the situation where the 1-bad arc is forced to arise appears highly unusual and places on the foliations a strange and strong restriction which may be ruled out by ad hoc considerations in many cases. It appears difficult to construct an example where this behavior occurs.

2. Main results. Our proof of 2.4 proceeds through several stages of gradual improvement. We shall be interested in the pattern made when two embedded discs in the compression body $M$ intersect. We introduce the following terminology. Suppose that $D_{1}$ and $D_{2}$ are two embedded discs in the compression body $M$ and arrange that they are in general position. Then $D_{1} \cap D_{2}$ is a union of simple closed curves and properly embedded arcs. By isotopies of the discs rel $\partial$, remove the former. We then define the dual tree of $D_{1} \cap D_{2}$ on $D_{i}$ by taking one vertex in each component of $D_{i} \backslash D_{1} \cap D_{2}$ and joining two vertices if they correspond to adjacent regions. Notice that the dual tree is not an isotopy invariant of $D_{1} \cup D_{2}$, that is, by isotoping $D_{2}$ rel , we may be able to change the dual tree on $D_{1}$. However this phenomenon will not concern us here since we shall be exclusively interested in avoiding the case of linear dual trees. So if some choice (rel d) of discs yields a nonlinear dual tree this will suffice.

We now fix our notation for the rest of this section. $\phi: F \rightarrow F$ will denote a pseudo-Anosov map with invariant foliations $\left(\mathscr{F}^{+}, \mu^{+}\right)$and $\left(\mathscr{F}^{-}, \mu^{-}\right)$. Our convention for the action of the map on these foliations will be the mnemonic one: positive powers of $\phi$ will stretch arcs with a positive superscript. Suppose that $\phi$ compresses in $M$. By abuse, we denote the extension of $\phi$ to the compression body by the same symbol.

Our first result is that a good arc may be obtained on at least one side. 
Proposition 2.1. There is an embedded disc $D$ in $M$, whose boundary $C=\alpha^{+} \cup \alpha^{-} \subset F$ satisfies

(i) $\alpha^{+}$and $\alpha^{-}$are bent arcs contained in $\mathscr{F}^{+}$and $\mathscr{F}^{-}$respectively.

(ii) At least one of $\alpha^{+}$or $\alpha^{-}$is good.

Proof. Theorem A provides a disc which satisfies all the hypotheses of the theorem, save possibly (ii). By choosing some power if necessary, we may assume that $\phi$ fixes the singularities and separatrices of the foliations. It is then easy to understand the action of the map $\phi$ along the bent arcs; it stretches the + arcs and shrinks the - arcs. In this way we see that for positive integers $m, n$ the intersections $\phi^{m}(C) \cap \phi^{-n}(C)$ lie in the + arc of $\phi^{m}(C)$ and the - arc of $\phi^{-n}(C)$, at least after a small general position push. We now claim that if the dual tree of $\phi^{m}(D) \cap \phi^{-n}(D)$ on either $\phi^{m}(D)$ or $\phi^{-n}(D)$ is nonlinear then we may prove the theorem. To fix our ideas, let us suppose that the dual tree is nonlinear on $\phi^{m}(D)$. Since $\phi^{m}(C)$ contains only two singularities and all intersections $\phi^{m}(C) \cap$ $\phi^{-n}(C)$ lie in the + arc, this implies that there is an outermost double arc $\gamma$ of $\phi^{m}(D) \cap \phi^{-n}(D)$ whose endpoints are joined by a regular + arc, $\beta^{+}$ say, of $\phi^{m}(C)$. The endpoints of $\gamma$ are also joined by a - arc, $\beta^{-}$say, of $\phi^{-n}(C)$ which may or may not be regular. The simple closed curve $\beta^{+} \cup \beta^{-}$bounds a disc in $M$, namely the one obtained by splicing the pieces of $\phi^{m}(D)$ and $\phi^{-n}(D)$ cut off by $\gamma$. Sliding the arc $\beta^{+}$transverse to the - foliation proves the result in this case.

So we are done unless for all positive integers $m, n$ the dual tree of $\phi^{m}(D) \cap \phi^{-n}(D)$ is linear on both $\phi^{m}(D)$ and $\phi^{-n}(D)$. Moreover, the two boundary vertices of the tree can always be taken at the singularities of the bent arcs.

Without loss of generality, (badness $\left.\beta^{+}\right) \leq$(badness $\beta^{-}$). Let $s$ be the singularity on $\beta^{+}$and $\partial \beta^{+}=\{p, q\}$. Since $\phi$ fixes the singularities and separatrices, $\phi^{m}\left(\beta^{+}\right)$lies along the arc $q s p$, although of course it is much longer. Let $b^{+}$and $b^{-}$be the innermost pair of intersections of $\phi^{-n}(C)$ with $\phi^{m}(C)$. Then $b^{+}$and $b^{-}$define a subarc of $\phi^{m}\left(\beta^{+}\right)$which contains $s$. Figure 3 shows the situation in the sector realising the badness of $\beta^{+}$. Then there is a double arc $\gamma$ of $\phi^{m}(D) \cap \phi^{-n}(D)$ with $\partial \gamma=\left\{b^{+}, b^{-}\right\}$, or we are done by the above argument. Thus we may orient $\phi^{-n}(C)$ so that at $b^{+}$the orientation points into the short sector, and at $b^{-}$it points out.

Let $c^{+}$be the first intersection of $\phi^{m}(C) \cap \phi^{-n}(C)$ following $b^{+}$on $\phi^{-n}(C)$ with this orientation. Similarly, let $c^{-}$be the first intersection preceding $b^{-}$. 


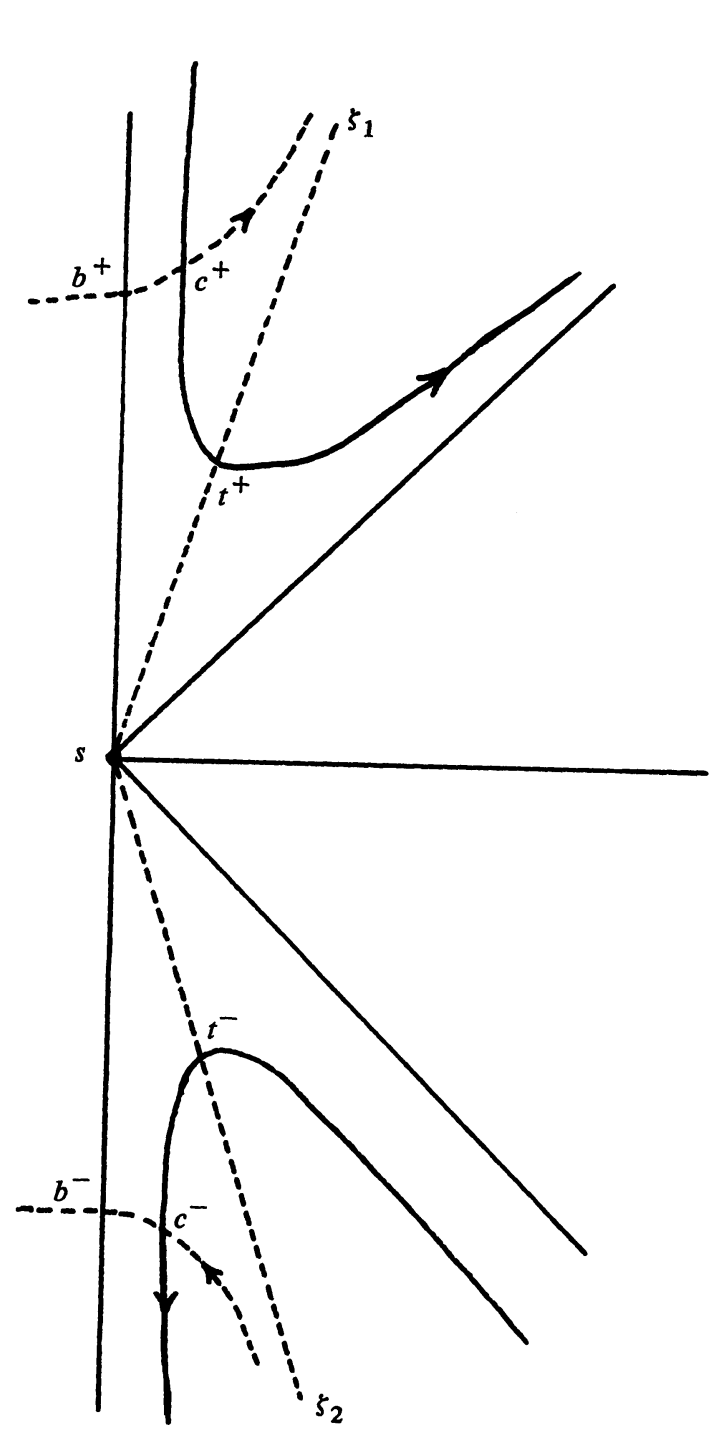

$g+$

$\mathscr{F}=$

FIGURE 3

Since the dual tree on $\phi^{-n}(D)$ was supposed to be linear, there is a double arc $\psi$ of $\phi^{m}(D) \cap \phi^{-n}(D)$ with $\partial \psi=\left\{c^{+}, c^{-}\right\}$. Hence we obtain the orientation shown on $\phi^{m}(C)$.

Consequently, choosing either arc of $\phi^{-n}(C)-\left\{c^{+}, c^{-}\right\}$together with either arc of $\phi^{m}(C)-\left\{c^{+}, c^{-}\right\}$gives a closed curve bounding a disc in $M$. A similar statement holds for $\left\{b^{+}, b^{-}\right\}$. Hence if we take the arc of $\phi^{m}(C)-\left\{c^{+}, c^{-}\right\}$which contains $b^{+}$(and so $\left.b^{-}\right)$we may 'short cut' 



Figure 4

across the $\operatorname{arc} b^{+} s b^{-}$to infer that either arc of $\phi^{m}(C)-\left\{c^{+}, c^{-}\right\}$, together with the arc $c^{+} b^{+} s b^{-} c^{-}$bounds a disc in $M$. (See Figure 4)

Observe that badness $\left(\beta^{+}\right) \leq$badness $\left(\beta^{-}\right)$and the fact that any selt intersection of $\phi^{-n}(C)$ at $s$ is inessential, combine to imply that neither of the separatrices of $\phi^{-n}\left(\beta^{-}\right)$lie in the sector around $s$ which we are considering. 
$\zeta_{1}$ and $\zeta_{2}$ be the two separatrices of $\mathscr{F}^{-}$through $s$ which are closest to $p s$ and $q s$ in the given sector. The remark of the previous paragraph shows that $\phi^{-n}\left(\beta^{-}\right)$does not lie along either of these. Let $t^{+}$be the first intersection of $\zeta_{1}$ on $\phi^{m}(C)$. Similarly, $t^{-}$is the first intersection of $\zeta_{2}$ on $\phi^{m}(C)$. Notice that $t^{+}$is immediately ahead of $c^{+}$on $\phi^{m}(C)$ by the innermost choice of $b^{+}$. Similarly, $t^{-}$immediately precedes $c^{-}$. Now observe that for $m, n$ large enough, the closed curves $s p b^{+} c^{+} t^{+} s$ and $s q b^{-} c^{-} t^{-} s$ both bound discs in the surface.

By hypothesis, $\partial \psi$ separates the singularities on $\phi^{m}(C)$ so there is a bent arc $\alpha^{+} \subset \phi^{m}(C)$ (lying entirely in $\mathscr{F}^{+}$) with $\partial \alpha^{+}=\left\{c^{+}, c^{-}\right\}$. Thus extending (or shortening) $\alpha^{+}$to a bent arc $\alpha$ we may arrange that $\partial \alpha=\left\{t^{+}, t^{-}\right\}$. Then the closed curve $\alpha \cup\left(\operatorname{arc} t^{+} s t^{-}\right)$is simple by choice of $t^{+}$and $t^{-}$. It is essential in $F$, since it is the union of two affine geodesics. Finally it is nullhomotopic in $M$ since it is homotopic in $F$ to the closed curve $\alpha^{+} \cup\left(\operatorname{arc} c b^{+} b^{+} s b^{-} c^{-}\right)$. This new simple closed curve has the further property that its badness on the + side is the same as that of $\beta^{+}$, while the badness on the - side is now badness $\left(\beta^{+}\right)-1$. By hypothesis this is strictly less than badness $\left(\beta^{-}\right)$. Hence the new curve is strictly better than the old one and an inductive argument completes the proof.

The above proof shows, somewhat surprisingly, that the difficult case in not the case of linear dual tree on both discs, but the case of a linear dual tree on one disc. Consequently in what follows we must assume that we are presented with asymmetric data, namely an infinitely persistent dual tree on one disc and the other pattern unknown.

The strategy will be to show that such situations happen very rarely and afford such control that we may make progress. The following lemma is a technical one; however it is of interest in its own right. It says that there is a disc in the compression which has the property that any three images of this disc under $\phi$ contain no essential triple points. This suggests that if a pseudo-Anosov extends, its foliations bound a geometric object 'like' a foliation by surfaces. This will be shown to be the true in the globally orientable case in $§ 3$.

Lemma 2.2. Let $\phi, F$ and $M$ be as above. Suppose further that $\phi$ fixes singularities and separatrices. Let $D$ be an embedded disc in $M$ whose boundary, $C$, is the union of two bent arcs. Let $m, n$ be any positive integers and by a small isotopy arrange that $\phi^{m}(C) \cap C \cap \phi^{-n}(C)$ is in general position. Then after an isotopy, rel $F$, of either $\phi^{m}(D)$ or $\phi^{-n}(D)$, the set $\phi^{m}(D) \cap D \cap \phi^{-n}(D)$ contains no triple points. 
Proof. Write $C=\alpha^{+} \cup \alpha^{-}$, two bent arcs. As in the proof of 2.1, we have that $\phi^{m}\left(\alpha^{+}\right) \supset \alpha^{+} \supset \phi^{-n}\left(\alpha^{+}\right)$and $\phi^{m}\left(\alpha^{-}\right) \subset \alpha^{-} \subset \phi^{-n}\left(\alpha^{-}\right)$. Therefore, the intersections of $\phi^{m}(C) \cap C$ occur in $\alpha^{-}$and the intersections of $\phi^{-n}(C) \cap C$ occur in $\alpha^{+}$. If we now look in $D$, we see that there are 'potentially' no triple points: that is, no double arc of $\phi^{m}(D) \cap D$ has endpoints separating the endpoints of any double $\operatorname{arc}$ of $\phi^{-n}(D) \cap D$. In such a situation, it is easy to remove triple points by isotopies rel $F$.

REMARK. There is a bound on the number and order of the singularities, depending only on the genus of $F$. Thus there is a $t$, so that $\phi^{t}$ satisfies the condition on singularities and separatrices.

A corollary of the lemma is that $\phi^{m}(D) \cap \phi^{m-1}(D) \cap \cdots \cap \phi^{-n}(D)$ contains no essential triple points for any $m, n$. Hence if we look in $D$ at the double arc pattern of $D \cap \phi^{m}(D)$, although this may vary as $m$ varies, no arc of $D \cap \phi^{m}(D)$ can cross an arc of $D \cap \phi^{m^{\prime}}(D)$. We shall exploit this limited amount of control to show that if a linear dual tree persists on one side as $m, n \rightarrow \infty$ then the behavior of the discs is of a special nature which we now describe.

Definition. Let $C, D$ be two essential, simple closed curves in general position on the surface $F$. Orient $C$ and $D$. Then $C \cap D$ is a collection of signed points. An equivalence relation on $C \cap D$ is said to be a pairing if the following two conditions are satisfied.

(i) Each equivalence class contains two points, of opposite sign.

(ii) Suppose that $x^{+}$and $x^{-}$are equivalent and $\alpha$ is any $\operatorname{arc}$ of $C$ or $D$ with $\partial \alpha=\left\{x^{+}, x^{-}\right\}$. Further suppose that there is a point $z^{+}$of $C \cap D$ lying in $\operatorname{int}(\alpha)$. Then the point equivalent to $z^{+}$also lies in int $(\alpha)$.

It is easy to see that a pair of simple closed curves admits a pairing if and only if there is a compression body in which $C$ and $D$ bound discs simultaneously. The 'only if' part is the pertinant one here; the pairing arises naturally from consideration of the double arcs of the discs which $C$ and $D$ bound. Bearing this in mind we introduce a further definition:

Definition. Suppose that $\alpha^{+}$and $\alpha^{-}$are bent arcs with $C=\alpha^{+} \cup \alpha^{-}$ a simple closed curve bounding a disc $D$ in $M$. As usual we shall assume that $\phi$ fixes the singularities and separatrices. Let $s$ be the singularity on $\alpha^{-}$. 
We say a pairing of $D \cap \phi^{m}(D)$ is parametrised by arc length along $\alpha^{-}$ if all intersections $x^{+}, x^{-} \subset C \cap \phi^{m}(C)$ which are paired satisfy $\mu^{+}\left(s x^{+}\right)$ $=\mu^{+}\left(s x^{-}\right)$, where $s x^{+}$denotes the subarc of $\alpha^{-}$with endpoints $s, x^{+}$.

REMARKS. (i) Notice in particular that this means $x^{+}$and $x^{-}$lie in different components of $\alpha^{-}-(s)$. In the case of ambiguity, caused by different choice of discs giving rise to different pairings, we shall say that $D \cap \phi^{m}(D)$ fails to be parametrised by arc length if the pairing arising from some choice of disc causes the property to fail.

(ii) The property of being parametrised by arc length would appear to be extremely unusual. In particular it implies an extremely regular distribution of intersecions along $\alpha^{-}$. In many cases we can rule this behavior out by an ad hoc examination of the foliations in question; however we have been unable to rule it out in general.

LEMMA 2.3. With the notation of the above definitions, suppose that either,

(a) For some $m>0, D \cap \phi^{m}(D)$ fails to be parametrised by arc length along $\alpha^{-}$, or

(b) The components of $\alpha^{-} \backslash\{S\}$ are not of equal length.

Then, for some $t>0, D \cap \phi^{t}(D)$ contains a double arc whose endpoints lie in the same component of $\alpha^{-} \backslash\{S\}$.

Proof. (a) If $D \cap \phi^{m}(D)$ fails to be parametrized by arc length along $\alpha^{-}$, then there is an arc of double points $\psi$ whose endpoints $a, b$ lie on $\alpha^{-}$ and have $\mu^{+}(s a) \neq \mu^{+}(s b)$. If $a$ and $b$ lie in the same component of $\alpha^{-}-\{s\}$ then we are finished, so we may suppose that this is not the case. For definiteness, say that $\mu^{+}(s a)>\mu^{+}(s b)$. Since as $k \rightarrow \infty$ the class of $\phi^{k}(\partial D)$ (considered via the operation of 'elargissement' to be a measured foliation) converges to $\left(\mathscr{F}^{+}, \mu^{+}\right)$, we have that for sufficiently large $t>0$,

$$
\left|\phi^{t}(\partial D) \cap s a\right|>\left|\phi^{t}(\partial D) \cap s b\right| .
$$

Consider $\phi^{m}(D) \cap D \cap \phi^{t}(D)$. By Lemma 2.2 and the remark following it, after an isotopy of $\phi^{t}(D) \operatorname{rel}(F)$, this set contains no triple points. But $\psi$ is a double arc joining $a$ and $b$, so no double arc of $\phi^{t}(D) \cap D$ can cross $\psi$. Looking in $D$ we see that (*) implies that there is a double arc of $\phi^{t}(D) \cap D$ with both endpoints in $s a$. This completes the proof.

(b) This has an analogous proof, taking $\{a, b\}=\partial \alpha^{-}$. 
We shall now prove the main theorem of this section:

THEOREM 2.4. Let $\phi: F \rightarrow F$ be a pseudo-Anosov automorphism, with invariant foliations $\left(\mathscr{F}^{+}, \mu^{+}\right),\left(\mathscr{F}^{-}, \mu^{-}\right)$of intercept length $K$. Suppose that $\phi$ compresses in $M$.

Then there is an embedded disc $D$ in $M$ whose boundary $\alpha^{+} \cup \alpha^{-} \subset F$ satisfies:

(i) $\alpha^{+}$and $\alpha^{-}$lie in $\mathscr{F}^{+}$and $\mathscr{F}^{-}$respectively and are bent arcs.

(ii) One of these bent arcs is good, the other is at worst 1-bad.

(iii) $\mu^{+}\left(\alpha^{-}\right), \mu^{-}\left(\alpha^{+}\right) \leq K$.

Proof. Proposition 2.1 guarantees $\beta^{+} \cup \beta^{-}=C$, which is a simple closed curve, good on one side and bounding an embedded disc in $M$. Without loss of generality, $\beta^{+}$is the good side. Let $s^{+}, s^{-}$be the singularity on $\beta^{+}, \beta^{-}$respectively. Our first aim is to find a disc satisfying (i) and (ii).

We claim that we are done if there is a $t>0$ so that $\phi^{t}(D) \cap D$ contains a double arc with both endpoints in the same component of $\beta^{-}-\left\{s^{-}\right\}$. For then the endpoints of this arc bound a regular $-\operatorname{arc}$ in $\beta^{-}$. On $\phi^{t}(C)$, these endpoints either separate the singularities, in which case they bound a good bent + arc, or they do not separate, in which case they bound a regular + arc. This proves the claim in either case.

Thus by 2.3 , we may assume that for all $t>0$ : (a) $D \cap \phi^{t}(D)$ is parametrised by arc length along $\beta^{-}$, (b) The tree on $D$ is linear, (c) The components of $\beta^{-}-\left\{s^{-}\right\}$have the same measure.

Application of $\phi$ now shows that for all positive integers $m$ and $n$, $\phi^{-n}(D) \cap \phi^{m}(D)$ has similar properties, namely that it is parametrised by arc length along $\phi^{-n}\left(\beta^{-}\right)$and has linear dual tree on $\phi^{-n}(D)$.

Let the endpoints of $\beta^{-}$be $b^{+}$and $b^{-}$. By Lemma 2.3(b), the arcs $s^{-} b^{-}$and $s^{-} b^{+}$have the same measure. Thus they are paired in $\phi^{-n}(D) \cap$ $\phi^{m}(D)$. This means that we may orient $\phi^{-n}(C)$ so that this orientation points out of the good sector at $b^{+}$and in at $b^{-}$. Let $a^{+}$be the first intersection of $\phi^{m}(C) \cap \phi^{-n}(C)$ following $b^{+}$on $\phi^{-n}(C)$ and $a^{-}$be the first one preceding $b^{-}$. By linearity $a^{+}$and $a^{-}$are paired. Let $\zeta_{1}$ and $\zeta_{2}$ be the two separatrices of $\mathscr{F}^{-}$passing through $s^{+}$which are closest to the arcs $s^{+} b^{+}$and $s^{+} b^{-}$, respectively. Let $c^{+}$be the first intersection of $\zeta_{1}$ with $\phi^{m}(C)$ and $c^{-}$the first of $\zeta_{2}$ with $\phi^{m}(C)$. Again, for $m, n$ sufficiently large, the closed curves $s^{+} c^{+} a^{+} b^{+}$and $s^{+} c^{-} a^{-} b^{-}$bound discs in the surface. See Figure 5 
$\mathscr{F}^{+}$
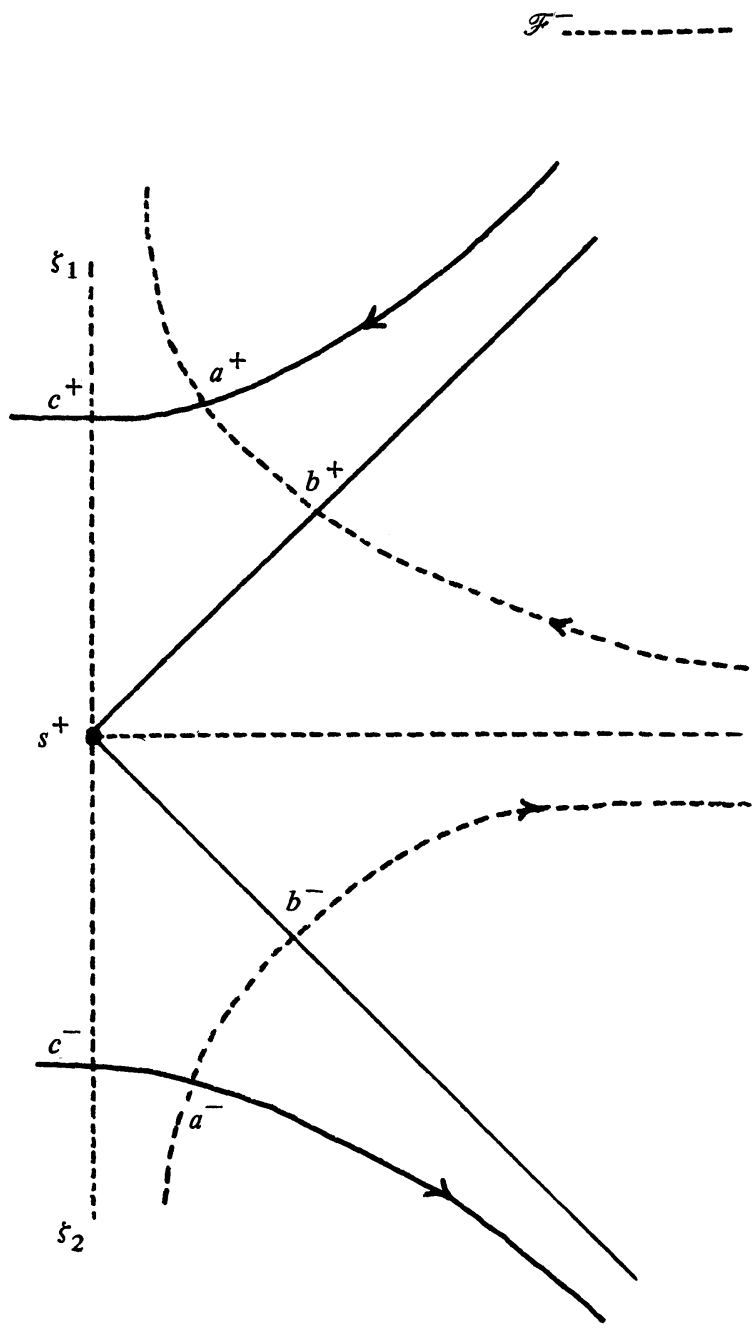

FIGURE 5

Now we apply a shortcircuiting trick analogous to the one used in 2.1 to conclude that the arc $c^{+} s^{+} c^{-}$together with either component of $\phi^{m}(C)-\left\{c^{+}, c^{-}\right\}$bounds a disc in $M$. The arcs $\beta^{+}$and $\beta^{-}$do not intersect the arc $c^{+} s^{+} c^{-}$by the innermost choice of $a^{+}, a^{-}$. Thus if we choose the component which contains $s^{+}$together with $c^{+} s^{+} c^{-}$, we obtain a simple closed geodesic (in the usual extended sense) bounding a disc in $M$, which has its - arc of badness 1 and + arc good. 
We have now achieved all the conclusions of the theorem, save (iii). We obtain this by applying the proof of [3] to the disc we have just manufactured. This never increases badness and gives the required control of lengths, completing the proof.

3. Applications. In this section we briefly explore some applications of the techniques and results of 2 .

The motivation for proving 2.4 was computational, however the methods show more than could have been anticipated a priori. For example, the possibilities in the event of there being no disc in the compression whose boundary consists of two good bent arcs are highly restricted, since the condition of being parametrised by arc length appears to be unusual and implies in particular that the 1-bad arc of any disc in the compression has to be bisected by its singularity. This raises the question of whether 2.4 is the best possible theorem. The following result suggests that perhaps it is.

TheOREM 3.1. Let $\phi: F \rightarrow F$ be a pseudo-Anosov whose invariant foliations are globally orientable. Then a compression of $\phi$ cannot contain a disc whose boundary is the union of two good bent arcs.

Proof. Recall that 'globally orientable' means that the foliations define a singular vector field on $F$. Then the only way that two good bent arcs can form a simple closed curve, $C$, is shown in Figure 6 . It is then easy to see that (possibly after iterating $\phi$ to fix the singularities and separatrices) that $C$ and $\phi^{k}(C)$ do not have zero algebraic intersection for large $k$.

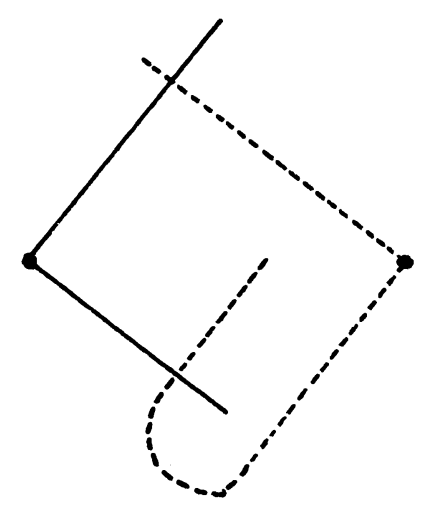

FIGURE 6 
It seems extremely difficult to construct a pseudo-Anosov with globally orientable foliations which compresses, but there appears to be no reason to expect that none exist. The proof of 2.4 shows that such a map has rather bizarre properties. Moreover there are several other results which may be obtained in the globally orientable case which do not obviously hold in the general case. We now derive some such results.

In order to state these, it is necessary to introduce some notation. It is well known the the universal covering of a compression body $p: M^{*} \rightarrow M$ induces a covering $p: F^{*} \rightarrow F$ for which the surface $F^{*}$ is planar, that is, it embeds in the 2-sphere. Typically, $F^{*}$ is the 2 -sphere with a cantor set removed. The foliations which we are considering have all their leaves contractible, so we may lift them to obtain a foliation of $F^{*}$. We shall say that a leaf in the lifted foliation is homoclinic if both ends of the leaf go to the same end of $F^{*}$. Equivalently, if we replace the cantor set, the leaf compactifies to become a simple closed curve. Our result may then be stated:

TheOREM 3.2. Let $\phi: F \rightarrow F$ be a pseudo-Anosov with globally orientable invariant foliations $\mathscr{F}^{+}$and $\mathscr{F}^{-}$. Suppose that $\phi$ compresses in $M$, giving rise to a planar covering $p: F^{*} \rightarrow F$. Then,

(a) Both lifted foliations have a homoclinic leaf.

(b) One of the lifted foliations has at least a countable number of its leaves homoclinic.

Proof. We shall prove (b). This will make it clear how to prove (a).

By Theorem 2.1, there is an embedded disc in $M$, whose boundary is the union of two bent arcs, one of which is good. Say that this is on the + side. The periodic points of a pseudo-Anosov are dense, hence by 'pushing off' on the good side (see Figure 7) we may find a simple closed curve $C=\alpha \cup \beta$, bounding a disc in $M$, and with $\alpha$ being a regular arc passing through a periodic point, $p$ say. Then $\phi^{k}$ fixes $p$ and so we may choose a lift of it which fixes some lift of $p$. The action of $\phi^{k}$ is to stretch $\alpha$ and it then describes the track of the lifted leaf through $p$. The Poincaré-Bendixson theorem (which extends to the singular vector fields we consider) guarantees that the lifted leaves are proper, that is, they do go to the ends. Since $\phi^{k}(C)$ lifts for all $k$ and $\phi^{k}(\beta)$ is getting very short as $k \rightarrow \infty$ we see that the lift of the leaf through $p$ is homoclinic. Thus, every lift of the leaf through $p$ is homoclinic. 


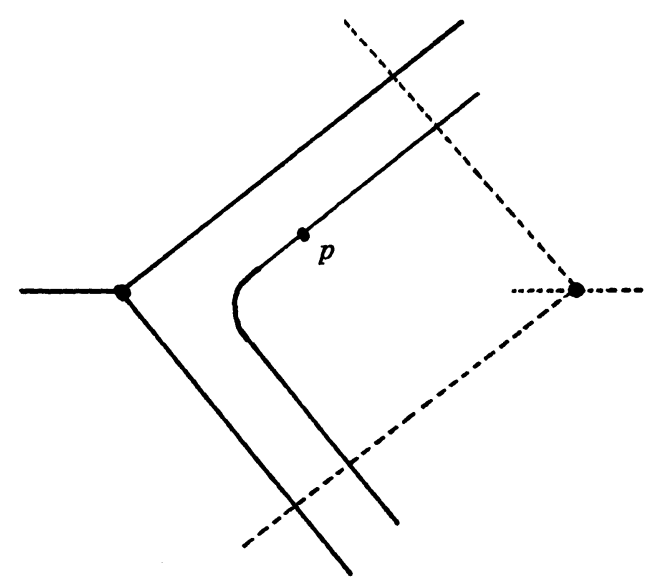

FIGURE 7

We have just shown that any leaf through a periodic point is homoclinic. Since a leaf can run through at most one periodic point, our argument shows that at least countably many leaves are homoclinic on the + side. This completes the proof.

Given this theorem, we may prove the following:

TheOREM 3.3. Let $\phi: F \rightarrow F$ be a pseudo-Anosov map with globally orientable foliations $\mathscr{F}^{+}$and $\mathscr{F}^{-}$. Suppose that $\phi$ compresses in a handlebody $M$. Then for one of the foliations we may find a collection of surfaces $\left\{S_{\alpha}\right\}$ embedded in $M$, such that:

(a) Given any regular leaf $\alpha$ in this collection, the embedded surface $S_{\alpha}$ has $\partial S_{\alpha}=\alpha$.

(b) If $\alpha$ and $\beta$ are two regular leaves, then $S_{\alpha}$ is disjoint from $S_{\beta}$.

Proof. Put a hyperbolic structure on $\operatorname{int}(M)$. Then this induces a conformal structure on $F$. By 3.2, one of the foliations has lifts to $F^{*}=\partial M^{*}$ homoclinic. Such lifted leaves, when compactified, look like simple closed curves in the boundary of hyperbolic space when this is identified with $M^{*}$. Thus we may form their 'convex hull.' The boundary of this convex hull is (in general) the union of two 'pleated surfaces'. (See [7].) Since the foliation is globally orientable, an orientation on $F$ and an orientation on the foliation give a choice of 'top' pleated surface which is consistent for the action of the group of covering translations.

If $\alpha^{*}$ is a lifted leaf which is homoclinic, and $g \alpha^{*}$ is any translate, then the compactifications of $\alpha^{*}$ and $g \alpha^{*}$ are either disjoint or meet in one point, the latter case only occurring when $g$ fixes the end which $\alpha^{*}$ goes to. 
Now observe that if two Jordan curves are disjoint, the 'top' surfaces of their convex hulls are disjoint. Similarly, if they meet in one point. Thus, either the top or bottom pleated surface projects into $M$ as an embedded surface. This proves (a). A similar argument gives (b).

REMARK. We do not know if this theorem continues to hold in the case that the foliation is only a line field. There are several problems with extending the above proof; we are unable to appeal to the PoincaréBendixson theorem and there may be no canonical choice of upper surface in the convex hull which is respected by the covering translations. It seems that the latter problem may be circumvented using the methods of minimal surfaces, however the possibility that the lifted leaves are not proper appears harder to overcome.

We conclude with a brief description of a series of examples of fibred knots for which these techniques seem particularly suitable. Let us remark that when applying these methods to knots, it is usual to work with the surface obtained by closing off the fibre surface with a disc and extending the monodromy by defining it to be the identity on this disc.

Our examples are the $p$-string, $q$-bight Turk's Head knots, $((p-1) \times q)^{*}$ in the notation of Conway. The ideas of Goldsmith [5] then give convenient representations of the (closed off) fibre surface and monodromy, by expressing the fibre as a branched covering over a sphere. In the special case, $p=3$, we obtain a covering $p: F_{q} \rightarrow S^{2}$, where the branching is over four points. It turns out that this induces on the fibre surface the structure of a 'branched flat' manifold (See [4] or [6]), and in this structure the foliations can be visualised. In this situation there is a $q$-symmetry and this can be used to show [6]:

THEOREM 3.4. Let $\phi: F_{q} \rightarrow F_{q}$ be the closed mondromy of the 3-string $q$-bight Turk's Head knot. Suppose that $\phi$ compresses in $M$ in such a way that there is no embedded disc in $M$ whose boundary is the union of two good, bent arcs. Then $q=5$ or 7 .

We shall not explain here how this is proved, and content ourselves with the remark that if $q=5$, the monodromy extends over a handlebody and a disc whose boundary is the union of two good bent arcs can be found. For $q=7$, we may use the above representation, together with the algorithm of [3] to show that there is no compression of the mondromy at 
all. It is at this stage that the full force of 2.4 is necessary-the singularities in the foliations of this knot have order 7. This shows via a theorem of Casson and Gordon [2] that the knot $14^{*}$ is not homotopically ribbon.

A little more maybe said when $q$ is even, for in this case the foliations are globally orientable and so by 3.1 the hypothesis that there is no disc whose boundary is the union of two good bent arcs is automatic. We deduce that none of these maps can compress and so none of these knots is ribbon. Actually, for Arf invariant reasons, none of these knots is slice, so this gives nothing new.

\section{REFERENCES}

[1] A. J. Casson, Automorphisms of surfaces after Nielsen and Thurston, Lecture Notes, University of Texas 1982.

[2] A. J. Casson and C. McA. Gordon, A loop theorem for duality spaces and fibred ribbon knots, Invent. Math., 74 (1983), 119-139.

[3] A. J. Casson and D. D. Long, Algorithmic compression of surface automorphisms, to appear in Invent. Math.

[4] A. Fathi, F. Laudenbach and V. Poenaru, Travaux de Thurston sur les surfaces, Asterisque, (1979), 66-67.

[5] D. Goldsmith, Symmetric fibred links. Knots, groups and 3-Manifolds, Ann. of Math. Studies 84, Princeton Univ. Press.

[6] D. D. Long, Cobordism of knots and surface automorphisms, Thesis, Cambridge University 1983.

[7] W. P. Thurston, Hyperbolic structures on 3-manifolds II: Surface groups and 3-manifolds which fiber over the circle, preprint.

Received August 20, 1984 and in revised form February 12, 1985.

UNIVERSITY OF SOUTHAMPTON

SOUTHAMPTON S09 5NH, ENGLAND 


\section{PACIFIC JOURNAL OF MATHEMATICS EDITORS}

\author{
V. S. VARADARAJAN (Managing Editor) \\ University of California \\ Los Angeles, CA 90024 \\ Hebert Clemens \\ University of Utah \\ Salt Lake City, UT 84112 \\ Charles R. DePrima \\ California Institute of Technology \\ Pasadena, CA 91125
}

R. FINN

Stanford University

Stanford, CA 94305

HermanN FlaschKa

University of Arizona

Tucson, AZ 85721

RAMESH A. GANGOLlI

University of Washington

Seattle, WA 98195

ROBION KIRBY

University of California

Berkeley, CA 94720

ASSOCIATE EDITORS
R. ARENS
E. F. BECKENBACH
B. H. NEUMANN
F. WOLF
K. YoshidA (1906-1982)

C. C. Moore

University of California

Berkeley, CA 94720

H. SAMELSON

Stanford University

Stanford, CA 94305

HAROLD STARK

University of California, San Diego

La Jolla, CA 92093

\section{SUPPORTING INSTITUTIONS}

UNIVERSITY OF ARIZONA

UNIVERSITY OF BRITISH COLUMBIA

CALIFORNIA INSTITUTE OF TECHNOLOGY

UNIVERSITY OF CALIFORNIA

MONTANA STATE UNIVERSITY

UNIVERSITY OF NEVADA, RENO

NEW MEXICO STATE UNIVERSITY

OREGON STATE UNIVERSITY
UNIVERSITY OF OREGON

UNIVERSITY OF SOUTHERN CALIFORNIA

STANFORD UNIVERSITY

UNIVERSITY OF HAWAII

UNIVERSITY OF TOKYO

UNIVERSITY OF UTAH

WASHINGTON STATE UNIVERSITY

UNIVERSITY OF WASHINGTON 


\section{Pacific Journal of Mathematics}

Vol. 122, No. $1 \quad$ January, 1986

Michael James Cambern, Near isometries of Bochner $L^{1}$ and $L^{\infty}$ spaces ....1 Kun Soo Chang, Gerald William Johnson and David Lee Skoug, The

Feynman integral of quadratic potentials depending on two time

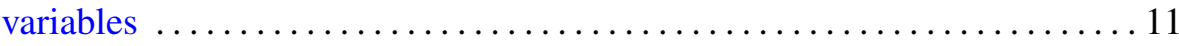

Robert Coleman, One-dimensional algebraic formal groups $\ldots \ldots \ldots \ldots \ldots 35$

Alberto Collino, The Abel-Jacobi isomorphism for the cubic fivefold .......43

N. J. Dev and S. S. Khare, Finite group action and vanishing of $N_{*}^{G}[F] \ldots 57$

Harold George Diamond and Jeffrey D. Vaaler, Estimates for partial sums

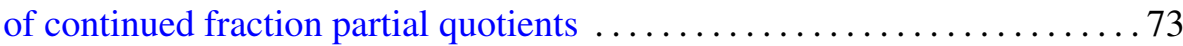

Kenneth R. Goodearl, Patch-continuity of normalized ranks of modules



Dean Robert Hickerson and Sherman K. Stein, Abelian groups and

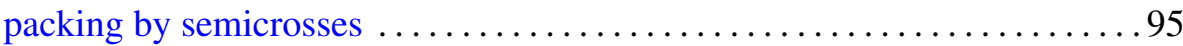

Karsten Johnsen and Harmut Laue, Fitting structures $\ldots \ldots \ldots \ldots \ldots 11$

Darren Long, Discs in compression bodies . ................... 129

Joseph B. Miles, On the growth of meromorphic functions with radially distributed zeros and poles ........................... 147

Walter Volodymyr Petryshyn, Solvability of various boundary value problems for the equation $x^{\prime \prime}=f\left(t, x, x^{\prime}, x^{\prime \prime}\right)-y \ldots \ldots \ldots \ldots \ldots . \ldots 169$

Elżbieta Pol, The Baire-category method in some compact extension problems

Masami Sakai, A new class of isocompact spaces and related results 211

Thomas Richard Shemanske, Representations of ternary quadratic forms and the class number of imaginary quadratic fields ..

Tsuyoshi Uehara, On class numbers of cyclic quartic fields 